\title{
Anticancer activity of bicalutamide-loaded PLGA nanoparticles in prostate cancers
}

\author{
JUN GUO $^{1 *}$, SHU-HONG WU²*, WEI-GUO REN ${ }^{3}$, XIN-LI WANG ${ }^{4}$ and AI-QING YANG ${ }^{5}$ \\ ${ }^{1}$ Department of Urology, The Affiliated Hospital of Taishan Medical University, Taian, Shandong 271000; \\ ${ }^{2}$ Department of Urology, Feicheng Kuangye Central Hospital, Feicheng, Shandong 271608; \\ ${ }^{3}$ Department of Urology, Rugao Boai Hospital, Nantong, Jiangsu 226500; ${ }^{4}$ Department of Pathology, \\ The Affiliated Hospital of Taishan Medical University; ${ }^{5}$ Department of Pathology, \\ The First People's Hospital of Taian, Taian, Shandong 271000, P.R. China
}

Received October 6, 2014; Accepted August 26, 2015

DOI: $10.3892 /$ etm.2015.2796

\begin{abstract}
Prostate cancer is the most commonly diagnosed non-cutaneous malignancy in men in western and most developing countries. Bicalutamide (BLT) is an antineoplastic hormonal agent primarily used in the treatment of locally advanced and metastatic prostate cancers. In the present study, the aim was to develop a nanotechnology-based delivery system to target prostate cancer cells. This involved the development of a BLT-loaded poly(D,L-lactide-co-glycolide) PLGA (PLGA-BLT) nanoparticulate system in an attempt to improve the therapeutic efficacy of BLT in prostate cancer and to mitigate its toxicity. Nanosized particles with a uniform size distribution and spherical shape were developed. PLGA-BLT showed a pronounced cytotoxic effect on LNCaP and C4-2 cancer cells. The superior cell-killing effect of the nanoparticles may be attributable to their sustained drug-release characteristics and high cellular internalization. PLGA-BLT was also found to significantly inhibit colony formation in the two cell lines. Furthermore, the caspase-3 activity of PLGA-BLT treated cancer cells was enhanced, indicating the cell apoptosis-inducing potential of PLGA-BLT. Overall, these results suggest that nanotechnology-based formulations of BLT exhibit superior anticancer activity and have enormous potential in the treatment of prostate cancers.
\end{abstract}

Correspondence to: Dr Wei-Guo Ren, Department of Urology, Rugao Boai Hospital, 468 Qingyu Road, Nantong, Jiangsu 226500, P.R. China

E-mail:weiguor@hotmail.com

*Contributed equally

Key words: prostate cancers, bicalutamide, anticancer activity, nanotechnology, poly(D,L-lactide-co-glycolide)

\section{Introduction}

Men in Western countries and developing nations are frequently diagnosed with cancers of the prostate gland (1). Statistically, prostate cancer has overtaken heart diseases and lung disorders in old people as a cause of mortality. According to estimates, $>200,000$ new cases of prostate cancer were reported with $>30,000$ mortalities during the year $2013(2,3)$. A similar or higher number of cases has been projected for the year 2014 (4). Mortality due to prostate cancer has declined in recent years; however, the expense of therapy remains very high (5). The treatment for prostate cancer mainly relies on chemotherapy, surgical intervention and ionizing radiation therapy. Conventional chemotherapy often results in low therapeutic effects with undesirable side-effects. The exposure of normal cells to anticancer drugs results in severe organ-related side-effects (6). Surgical interventions have a risk of reoccurrence and pain to the patient. Radiation therapy also results in adverse effects on rapidly dividing healthy cells (7). Therefore, a therapeutic drug delivery system that can enhance the chemotherapeutic efficacy of an anticancer drug while reducing its organ-related toxicity is highly desired.

Nanomedicine-based therapeutic approaches have been gaining increasing popularity. Nanotechnology-based delivery systems have attracted the attention of researchers from across the globe due to their tumor-targeting ability (8). This type of drug delivery system provides the sustained release of anticancer drug to target cancer cells and thereby avoids the exposure of normal cells. This unique approach can greatly enhance the therapeutic outcome while reducing drug-related severe toxicity (9). In this regard, several researchers have successfully demonstrated the potential of a nanomedical approach in the treatment of various cancers. In optimal cases, it decreases the mortality and morbidity rates associated with the cancers. Among various nanoparticulate delivery systems (lipid nanoparticles, nanomicelles, inclusion complexes, nanosuspensions and liposomes), biodegradable polymer-based nanoparticles have shown enormous potential for use in cancer drug delivery (10-12). Specifically, the polylactide (PLA)- and polyglycolide (PGA)-based polymer known as poly(D,L-lactide-co-glycolide) (PLGA) has been 
extensively studied owing to its biocompatible, biodegradable, non-toxic, non-immunogenic and noncarcinogenic properties $(13,14)$. PLGA-based nanomedicine products are being investigated in different phases of clinical trials (15-17). In particular, a docetaxel-loaded PLGA formulation has completed phase I clinical trial for the treatment of prostate cancer (4). Such proof-of-success inspired the present study in which an anticancer drug was encapsulated in PLGA nanoparticles to obtain a potential therapeutic for prostate cancer.

Bicalutamide (BLT), a nonsteroidal antiandrogen, was selected in the present study as a model drug for incorporation into PLGA nanoparticles in order to investigate its therapeutic effect in prostate cancers. BLT is an FDA-approved antineoplastic hormonal agent primarily used in the treatment of prostate cancer (18). It is widely used in the treatment of locally advanced and metastatic prostate cancer, either as a monotherapy or combined with other anticancer agents. It acts primarily by inhibiting the binding of androgen with androgen receptors. Moreover, BLT is a biopharmaceutics classification system (BCS) class II drug with low aqueous solubility and high permeability (19). The incorporation of BLT into PLGA nanoparticles should improve its dispersity and aqueous solubility, making it ideal for cancer drug delivery.

The present study aimed to develop a BLT-loaded PLGA nanoparticulate system in order to improve the therapeutic efficacy of BLT in prostate cancer and to mitigate its toxicity. The drug was loaded into PLGA nanoparticles and its size distribution and release characteristics were evaluated. The morphology of the nanoparticles was observed using a transmission electron microscope (TEM). The cytotoxic potential of free BLT and PLGA-BLT was studied by MTT assay, and a caspase-3 assay was carried out to investigate the apoptosis of cancer cells. Finally, the inhibitory effects of the drug-loaded formulation on cell growth and colony formation were studied in two prostate cancer cell lines. The LNCaP and C4-2 cell lines were selected as androgen-dependent and androgen-independent cells.

\section{Materials and methods}

Materials. PLGA (molecular weight, 25,000) was purchased from Sigma-Aldrich (St. Louis, MO, USA). BLT was procured from Beijing Guolian Chenhui Pharmaceutical Technology Co., Ltd. (Beijing, China). C4-2 human prostate cancer cells and lymph node prostate adenocarcinoma ( $\mathrm{LNCaP})$ cells were purchased from American Type Culture Collection (ATCC; Manassas, VA, USA). All other chemicals were of reagent grade and used without further modifications.

Preparation of BLT-loaded PLGA nanoparticles. BLT-loaded PLGA nanoparticles were prepared by a nanoprecipitation method. In brief, $5 \mathrm{mg}$ BLT and $50 \mathrm{mg}$ PLGA were dissolved in $10 \mathrm{ml}$ acetone and dissolved by vortexing and sonication. To this solution, $0.5 \%$ poloxamer 407 solution (Sigma-Aldrich, St. Louis, MO, USA) (10 ml) was added drop-by-drop in a controlled manner. The solution was stirred at a high speed in a magnetic stirrer and kept overnight at room temperature. Following evaporation of the organic solvents, drug-loaded nanoparticles were collected by centrifuging at 5,000 $\mathrm{x} g$ for
$20 \mathrm{~min}$ at $37^{\circ} \mathrm{C}$. The supernatant was removed and the pellet was re-dispersed with water and stored until use.

Particle size analysis. The particle size and size distribution of the PLGA-BLT nanoparticles were determined by a laser diffraction technique. A Malvern Zetasizer (Nano-ZS; Malvern Instruments, Malvern, UK) was employed to measure the size characteristics. The samples were diluted with double distilled water and the particle size was evaluated in triplicate. All measurements were performed at a fixed angle of $90^{\circ}$ at $25^{\circ} \mathrm{C}$ room temperature. The results were expressed as the mean size \pm standard deviation (SD).

Morphology. The morphological examination of PLGA-BLT was carried out using a TEM (Hitachi ${ }^{\circledR}$ 800-MT; Hitachi, Tokyo, Japan). The liquid samples were counterstained with phosphotungstic acid, then placed over a carbon-coated copper grid and air-dried.

Loading efficiency. The amount of drug loaded into the nanoparticles was evaluated by calculating the amount of drug added and the amount entrapped in the nanoparticles. For the latter, BLT-loaded PLGA nanoparticles were combined with $\mathrm{HCl}$ and stirred for $24 \mathrm{~h}$. The mixture was centrifuged $8,000 \mathrm{x} \mathrm{g}$ for 20 min at $37^{\circ} \mathrm{C}$, and the supernatant was collected to analyze the BLT via high-performance liquid chromatography (HPLC) using an LC 1200 system (Agilent Technologies, Santa Clara, CA, USA) with a reverse-phase $\mathrm{C} 18$ column $(250 \times 4.6 \times 5 \mathrm{~mm})$ at $25 \mathrm{C}$. The entrapment efficiency was calculated using the following formula: Entrapment efficiency $(\%)=$ amount of BLT in PLGA nanoparticles/total amount of BLT x 100.

Release study. The release of BLT in phosphate-buffered saline (PBS) and acetate-buffered saline (ABS) was evaluated by a dialysis method. For this purpose, $1 \mathrm{ml}$ PLGA-BLT suspension, suspended in release buffer and containing $1 \mathrm{mg}$ BLT, was transferred into a dialysis tube (molecular weight cutoff, 3,000; Membra-Cel ${ }^{\circledR}$; Viskase, Darien, IL, USA), sealed, and placed in a tube containing $20 \mathrm{ml}$ release medium. The whole assembly was kept in a horizontal laboratory shaker $(80 \mathrm{x} \mathrm{g})$ and maintained at $37^{\circ} \mathrm{C}$. At specific time intervals, $1 \mathrm{ml}$ sample was removed and replaced with equal amount of fresh release medium. The amount of drug present in the release media was analyzed using a HPLC method. The mobile phase consisted of PBS (pH 3.0 adjusted with phosphoric acid) and acetonitrile in a 55:45 v/v ratio. The mobile phase was filtered using a $0.45-\mu \mathrm{m}$ filter, degassed and run at a rate of $1.1 \mathrm{ml} / \mathrm{min}$. BLT was detected using a 112 UV detector (Gilson, Middleton, WI, USA) at $270 \mathrm{~nm}$.

Cell culture. C4-2 and LNCaP prostate cancer cells were cultured in RPMI-1640 medium (Sigma-Aldrich), which was supplemented with $10 \%$ fetal bovine serum (FBS), $1 \%$ penicillin-streptomycin mixture and $1 \%$ sodium pyruvate. The cells were incubated in ambient conditions of $5 \% \mathrm{CO}_{2}$ at $37^{\circ} \mathrm{C}$.

Cytotoxicity assay. The cytotoxic potentials of free BLT and PLGA-BLT were studied by 3-(4,5-dimethylthiazol-2-yl)-2,5-diphenyltetrazolium bromide (MTT) assay. C4-2 and LNCaP cells were seeded in a 96-well plate at a 
A

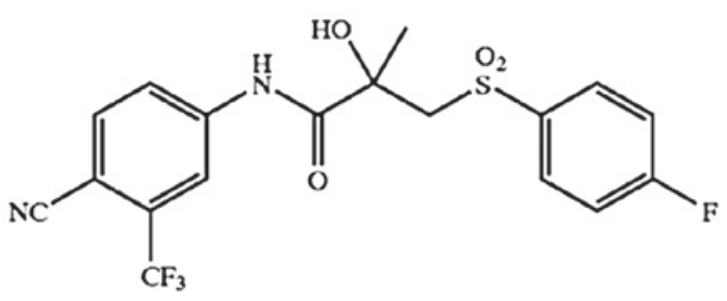

B

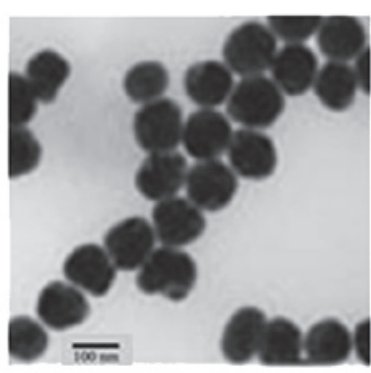

C

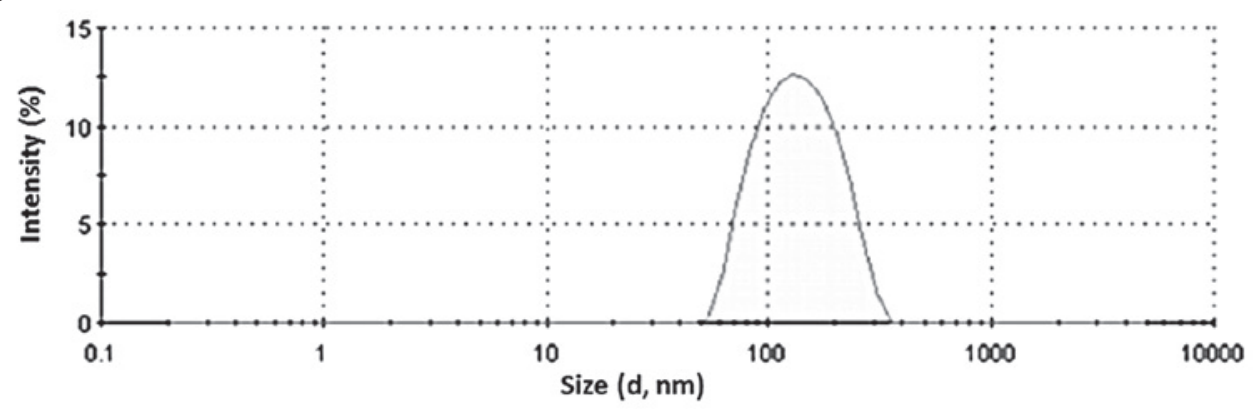

Figure 1. (A) Molecular structure of bicalutamide (BLT). (B) Transmission electron microscope image of PLGA-BLT nanoparticles. (C) Particle size distribution of PLGA-BLT nanoparticles (scale bar, $100 \mathrm{~nm}$ ). Particle size distribution was observed using laser diffraction. PLGA, poly(D,L-lactide-co-glycolide).

seeding density of $4 \times 10^{3}$ cells/well and were allowed to attach for $48 \mathrm{~h}$ until they reached $90 \%$ confluency. The cells were treated with free BLT or PLGA-BLT and further incubated for $24 \mathrm{~h}$ at concentrations of $0.1,0.5,1,5,10$ and $50 \mu \mathrm{g} / \mathrm{ml}$. The drug-containing media were removed carefully and replaced with fresh growth media containing MTT solution (0.5 mg), and the cells were then incubated for additional $3 \mathrm{~h}$. The supernatant was discarded carefully and $100 \mu 1$ DMSO was added to extract the formazan crystals. The intensity of the purple coloration was measured using a SpectraMax ${ }^{\circledR}$ Plus 384 UV-Visible microplate reader (Molecular Devices, Sunnyvale, CA, USA) at a wavelength of $570 \mathrm{~nm}$.

Caspase-3 activity. Caspase-3 activity of the C4-2 and LNCaP cells was analyzed using a Caspase-Glo 3 assay kit according to the instructions provided by the manufacturer (Promega, Madison, WI, USA). Cells were seeded in a 12-well plate at a density of $2 \times 10^{5}$ cells/well, treated with $5 \mu \mathrm{g}$ free BLT or an equivalent amount of PLGA-BLT and incubated for $24 \mathrm{~h}$ at $37^{\circ} \mathrm{C}$. Cells were washed with PBS and harvested using $0.25 \%$ trypsin. The cells were centrifuged at $500 \mathrm{x} \mathrm{g}$ for $30 \mathrm{sec}$ at $37^{\circ} \mathrm{C}$, re-dispersed and treated with $100 \mu \mathrm{l}$ Caspase-Glo and incubated for $1 \mathrm{~h}$. The solutions were transferred to culture tubes and examined using a luminometer (Lumat3 LB 9508; Berthold Technologies GmbH \& Co. KG, Bad Wildbad, and Germany).

Clonogenic assay. C4-2 and LNCaP cells were seeded in a 6 -well plate at a density of 500 cells/well. The cells were allowed to form colonies for 3 days and then treated with 1,5 and $10 \mu \mathrm{g} / \mathrm{ml}$ free BLT or PLGA-BLT for a week. The drug-containing media were removed and replaced with fresh growth media and the cells were further incubated for 2 weeks. The cells were washed twice with PBS, fixed, and stained with hematoxylin. The colonies were observed using a MultiImage ${ }^{\mathrm{TM}}$ light cabinet (Alpha Innotech Corporation, San
Leandro, CA, USA) with the aid of AlphaEaseFC ${ }^{\text {TM }}$ (Alpha Imager HP automatic image capture) software. The number of colonies was counted and quantified using a stereomicroscope (SMZ745; Nikon Corporation, Tokyo, Japan).

Statistical analysis. Statistical significance was analyzed using Student's t-test for two groups and one way analysis of variance for multiple groups. The level of significance was set at $\mathrm{P}<0.05$. All the studies were performed in triplicate and data are presented as the mean $\pm \mathrm{SD}$.

\section{Results and Discussion}

The treatment options for prostate cancer that are commonly used at present are chemotherapy, surgical intervention and ionizing radiation therapy. However none of these therapeutic approaches effectively controls tumor proliferation, with 20-30\% reoccurrence rates in patients (1-3). In this regard, BLT, an FDA-approved antineoplastic hormonal agent is primarily indicated for the treatment of prostate cancer (Fig. 1A). It is widely used in the treatment of locally advanced and metastatic prostate cancer, either as a monotherapy or in combination with other anticancer agents. However, the clinical translation of BLT faces numerous challenges, since the drug has poor pharmacokinetics and insufficient aqueous solubility (20). Therefore, the incorporation of BLT into a nanoparticulate system is expected to improve its therapeutic efficacy towards prostate cancers. The aim of the present study was to evaluate the anticancer effects of free BLT and PLGA-BLT in androgen-dependent and androgen-independent prostate cancer cell lines. PLGA has been extensively studied owing to its biocompatible, biodegradable, non-toxic, non-immunogenic and noncarcinogenic properties (13-15). PLGA-based nanomedicine products are being evaluated in clinical trials (16). Inspired by the clinical success of PLGA nanocarriers, a PLGA polymer was employed to encapsulate 


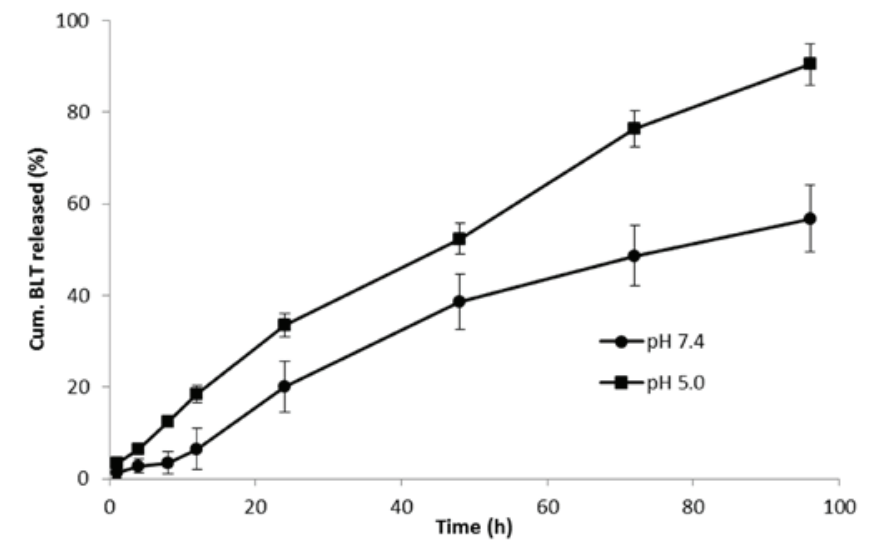

Figure 2. In vitro release study of PLGA-BLT nanoparticles. The release study was performed in phosphate-buffered saline and acetate-buffered saline at $37^{\circ} \mathrm{C}$. The amount of drug released was estimated using HPLC. PLGA, poly(D,L-lactide-co-glycolide); BLT, bicalutamide; HPLC, highperformance liquid chromatography; cum, cumulative.

BLT in the present study. In addition, BLT is hydrophobic with poor aqueous solubility, making it difficult to administer intravenously. The loading of BLT into PLGA nanoparticles is likely to improve its systemic characteristics and physicochemical properties.

Physicochemical analysis of PLGA-BLT. A nanoprecipitation method was employed to prepare the BLT-loaded PLGA nanoparticles. As shown in Fig. 1B, the particles were spherical in shape with a dense core complex. When observed under a TEM, the mean size of the particles was found to be $\sim 100 \mathrm{~nm}$ with a uniform distribution of the particles on the surface of the TEM grid (Fig. 1B). No aggregation or distortion of the nanoparticles was observed during the dry state analysis. Furthermore, size was further confirmed with a dynamic light scattering (DLS) technique using laser diffraction. This revealed a uniform size distribution with an average size between 100 and $120 \mathrm{~nm}$ (Fig. 1C). It should be noted that the TEM measured the particle in a dried state while DLS measured the particle in a hydrodynamic state. A particle size of $\sim 100 \mathrm{~nm}$ should be ideal for cancer drug delivery (21). A nanosized particle is able to take advantage of passive targeting and the enhanced permeability retention (EPR) effect.

Release study. The release of BLT from the PLGA nanoparticles was studied in PBS and ABS at $37^{\circ} \mathrm{C}$. As shown in Fig. 2, BLT presented a sustained-release profile at the two $\mathrm{pH}$ conditions. No initial burst release phenomenon was observed at any point of the study period. At $\mathrm{pH} 7.4$, the drug was released in a sustained manner and nearly $60 \%$ of the drug was released by the end of $96 \mathrm{~h}$. Accelerated drug release was observed at $\mathrm{pH}$ 5.0, indicating the $\mathrm{pH}$-responsive behavior of the PLGA nanocarrier. At lower $\mathrm{pH},>90 \%$ of the drug was released. The sustained release of the drug in the region of a tumor should increase the chemotherapeutic efficiency of the anticancer drug. Moreover, it can be expected that the delivery system will protect healthy cells from exposure to the drug and provide a constant dose level to cancer cells. It should be noted that the release of the drug from the nanoparticle matrix may occur either by drug diffusion or by erosion of the nanoparticle

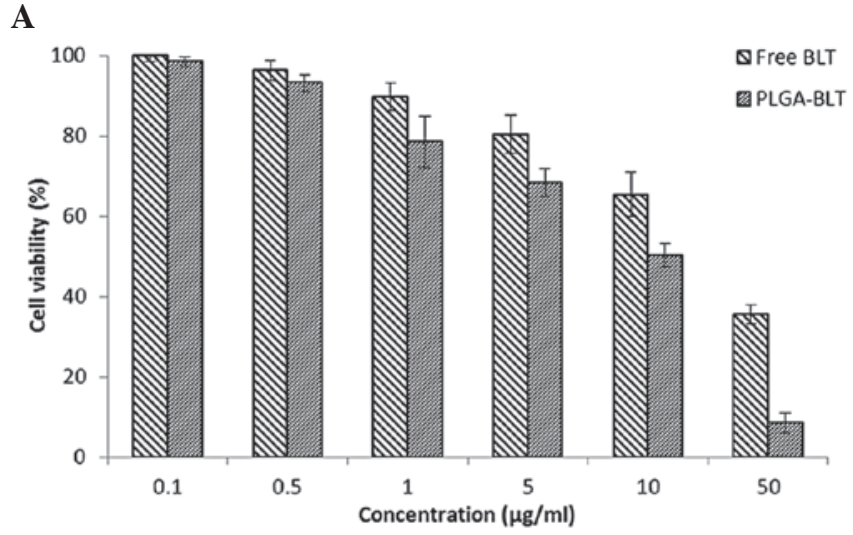

B

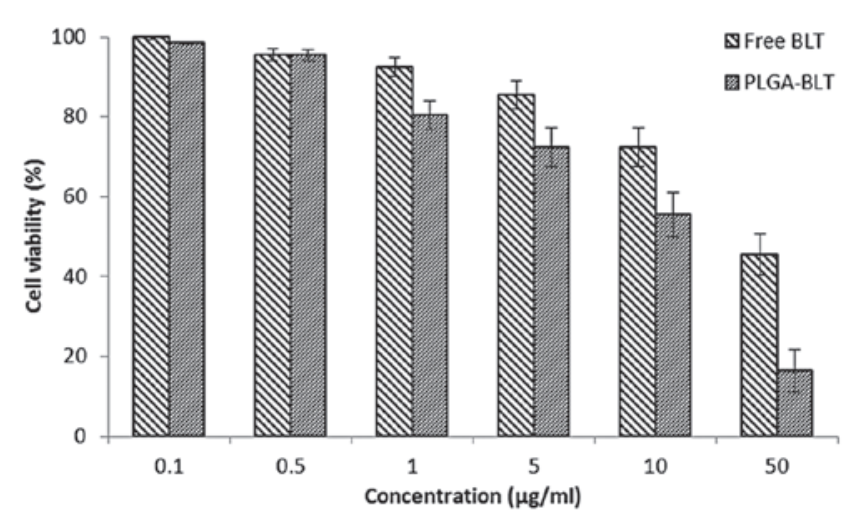

Figure 3. In vitro cytotoxicity of free BLT and PLGA-BLT nanoparticles to (A) LNCaP and (B) C4-2 prostate cancer cells. The cell cytotoxicity was evaluated by MTT assay with measurement of the intensity of coloration at $570 \mathrm{~nm}$. The study was performed with various concentration of the two drug formulations with treatment for $24 \mathrm{~h}$. MTT, 3-(4,5-dimethylthiazol-2-yl)-2,5-diphenyltetrazolium bromide; BLT, bicalutamide; PLGA, poly(D,L-lactide-co-glycolide).

architecture. Slow release of the drug in this manner upon systemic administration should provide constant exposure of the cancer cells to the drug, thereby enhancing the cytotoxic effect. Moreover, sustained release is likely to avoid unnecessary drug-related side-effects in normal organs of the body.

In vitro cell viability. The cytotoxic potential of PLGA-BLT and free BLT in LNCaP (androgen dependent) and C4-2 (androgen independent) cancer cells was then investigated. The cell viability of each cell line was evaluated using MTT assay. Cells were treated with increasing concentrations of drug ranging from 0.1 to $50 \mu \mathrm{g} / \mathrm{ml}$. As shown in Fig. 3, the drug-loaded formulation had a concentration-dependent cytotoxic effect on cell proliferation. PLGA-BLT exhibited a pronounced cell killing effect comparing with that of free BLT. Another important observation is that neither BLT nor PLGA-BLT were effective at lower concentrations while at higher concentrations $(>10 \mu \mathrm{g} / \mathrm{ml})$ they demonstrated significant cytotoxicity. In order to quantify the effect of the two formulations, the $\mathrm{IC}_{50}$ value of each was calculated. The $\mathrm{IC}_{50}$ values of PLGA-BLT in LNCaP and C4-2 cells were 45.4 and $58.5 \mu \mathrm{g} / \mathrm{ml}$, respectively. However, free BLT exhibited a higher $\mathrm{IC}_{50}$ value in the two cancer cell lines (48.9 and 65.8 $\mu \mathrm{g} / \mathrm{ml}$ ) respectively. The results, therefore, clearly showed that nanocarrier-based BLT remarkably enhanced the therapeutic 
A

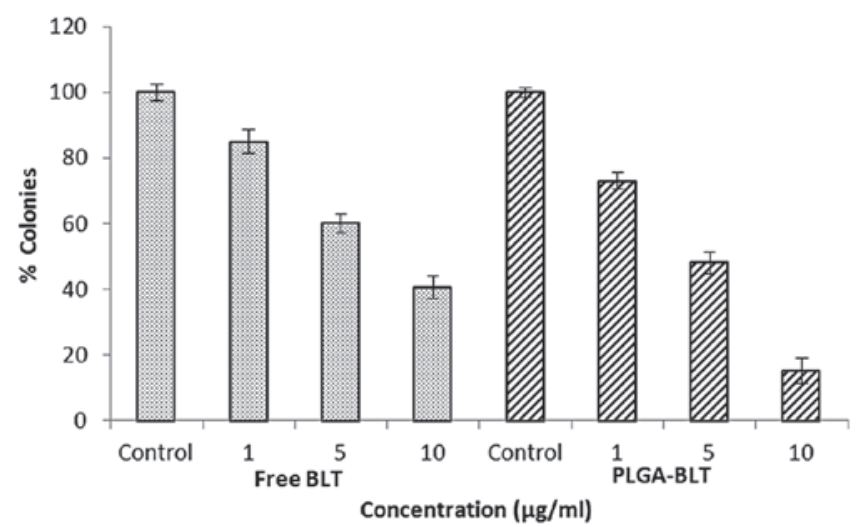

B

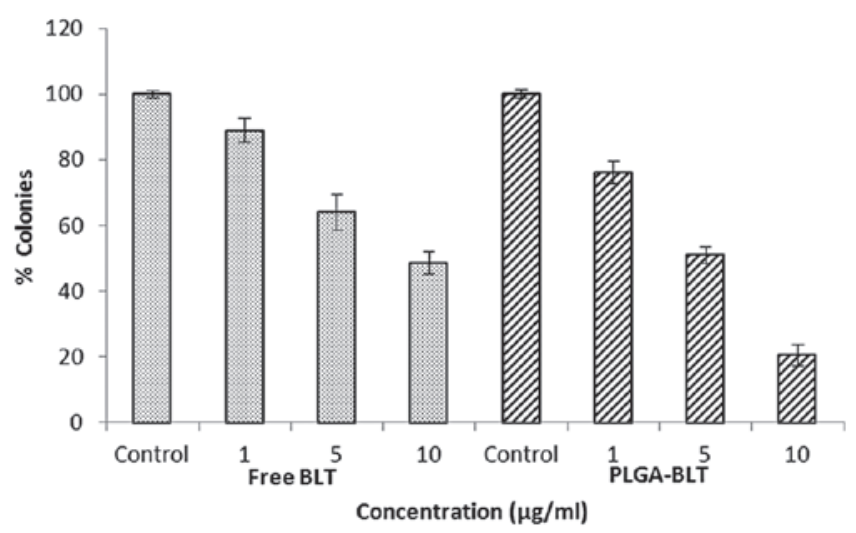

Figure 4. Effect of free BLT and PLGA-BLT nanoparticles on the clonogenic potential of (A) LNCaP and (B) C4-2 prostate cancer cells. The number of colonies was quantified using an automatic image capture software analysis tool. BLT, bicalutamide; PLGA, poly(D,L-lactide-co-glycolide).

or anticancer effect of the drug. The superior cytotoxicity of PLGA-BLT may be attributed to its sustained-release characteristics and higher cellular internalization, whereas the free drug enters cells by passive diffusion (22).

Clonogenic assay. The anticancer effects of the free drug and drug-loaded formulation were also determined by evaluation in a clonogenic assay. Untreated cells produced large colonies while drug-treated cells showed markedly fewer colonies. The colony-forming effect was studied in the presence of increasing concentrations of the drug. As shown in (Fig. 4), the colony-forming ability of the cells was inhibited by the two formulations in a strictly dose-dependent manner. Specifically, PLGA-BLT demonstrated a greater inhibitory effect on colony formation than was observed for free BLT. At all concentrations, the two drug formulations inhibited the colony forming ability of the cells. Although, BLT at a concentration of $1 \mu \mathrm{g} /$ $\mathrm{ml}$ showed mild inhibitory effects on colony formation, marked inhibition was observed at a concentration of $10 \mu \mathrm{g}$ / ml. Notably, at each concentration, the drug-loaded nanocarriers induced a prominent reduction in colony formation. Although a slight difference in inhibitory level was observed between the two cell lines, the trend observed in the cell lines was similar. Blockage of androgen activity does not have high efficiency in C4-2 cells, which are androgen independent. Overall, the results suggest that the slow and sustained release
A

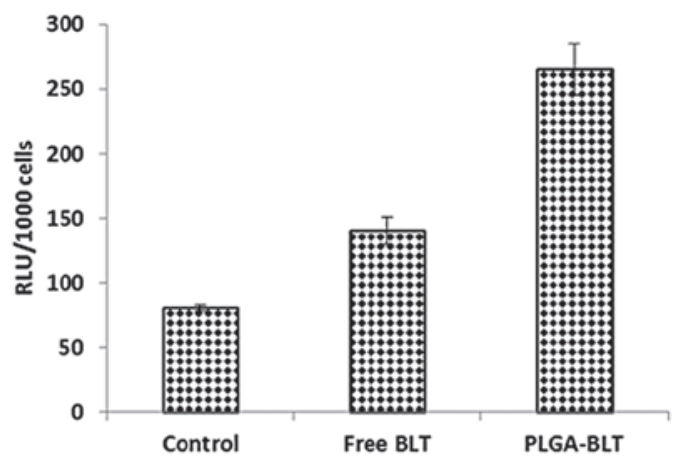

B

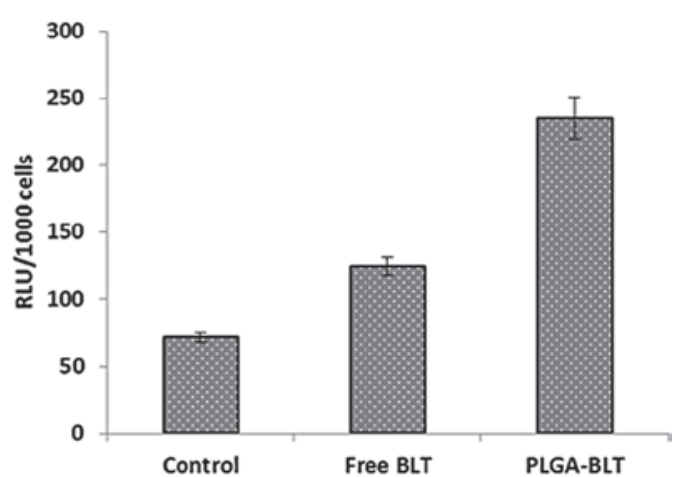

Figure 5. Caspase-3 activity of free BLT and PLGA-BLT nanoparticles in (A) $\mathrm{LNCaP}$ and (B) $\mathrm{C} 4-2$ prostate cancer cells. The cells were treated with free BLT or PLGA-BLT for $24 \mathrm{~h}$ and caspase-3 activity was measured using a luminescent assay kit. The intensity of the emitted light was measured using a luminometer. BLT, bicalutamide; PLGA, poly(D,L-lactide-co-glycolide); RLU, relative light unit.

of BLT from PLGA nanoparticles resulted in PLGA-BLT exhibiting a superior performance compared with free BLT. This indicates that the overall performance of BLT may be improved by incorporating it into nanoparticles.

Apoptotic activity by caspase-3 assay. The superior cell inhibitory effect of PLGA-BLT was further confirmed by the evaluation of caspase-3 activity (Fig. 5). Significantly higher caspase-3 activity was observed in the PLGA-BLT-treated cells compared with that in the cells treated with free BLT. High caspase- 3 activity is an indication of a greater degree of cell apoptosis (23). This result is consistent with the marked cytotoxic potential and growth inhibitory effect of the PLGA-BLT nanoparticles on colony formation.

Conclusion. BLT-loaded PLGA nanoparticles were successfully prepared and their anticancer effect evaluated in LNCaP and C4-2 cancer cells. Nanosized PLGA-BLT particles with a uniform size distribution and spherical shape were developed. The PLGA nanoparticles released BLT in a slow and sustained manner, such that cancer cells should be exposed to a constant level of the drug. The PLGA-BLT nanoparticles showed a pronounced cytotoxic effect on LNCaP and C4-2 cancer cells. The superior cell killing effect of PLGA-BLT nanoparticles compared with that of free BLT may be due to the sustained drug release characteristics and high cellular internalization of 
the particles. In addition, PLGA-BLT has been demonstrated to significantly inhibit the colony formation activity of the two cell lines. Finally, the caspase-3 activity of PLGA-BLT-treated cancer cells has been indicated to be enhanced compared with that of cancer cells treated with free BLT, indicating the cell apoptosis-inducing potential of PLGA-BLT. Overall, these results suggest that nanotechnology-based BLT treatment should be able to effectively target and kill cancer cells. This study may pave the way for the successful chemotherapeutic treatment of prostate cancers.

\section{Acknowledgements}

The study was supported by a University Grant allocated for research.

\section{References}

1. Siegel R, Ma J, Zou Z and Jemal A: Cancer statistics, 2014 CA Cancer J Clin 64: 9-29, 2014.

2. Gianino MM, Galzerano M, Minniti D, Di Novi C, Martin B, Davini $\mathrm{O}$ and Barbaro S: A comparative costs analysis of brachytherapy and radical retropubic prostatectomy therapies for clinically localized prostate cancer. Int J Technol Assess Health Care 25: 411-414, 2009.

3. Snyder CF, Frick KD, Blackford AL, Herbert RJ, Neville BA, Carducci MA and Earle CC: How does initial treatment choice affect short-term and long-term costs for clinically localized prostate cancer? Cancer 116: 5391-5399, 2010.

4. Li J, Djenaba JA, Soman A, Rim SH and Master VA: Recent trends in prostate cancer incidence by age, cancer stage, and grade, the United States, 2001-2007. Prostate Cancer 2012: 69138, 2012

5. Niraula S and Tannock IF: Broadening horizons in medical management of prostate cancer. Acta Oncol 50 (Suppl 1): $141-147,2011$

6. Sowery RD, So AI and Gleave ME: Therapeutic options in advanced prostate cancer: Present and future. Curr Urol Rep 8: 53-59, 2007.

7. Mishra B, Patel BB and Tiwari A: Colloidal nanocarriers: A review on formulation technology, types and applications toward targeted drug delivery. Nanomedicine 6: 9-24, 2010.

8. Nishiyama $\mathrm{N}$ and Kataoka K: Current state, achievements and future prospects of polymeric micelles as nanocarriers for drug and gene delivery. Pharmacol Ther 112: 630-648, 2006.

9. Torchilin VP: Micellar nanocarriers: Pharmaceutical perspectives. Pharm Res 24: 1-16, 2007.
10. Murthy RS and Umrethia ML: Optimization of formulation parameters for the preparation of flutamide liposomes by 3(3) factorial 26-term logit model. Pharm Dev Technol 9: 369-377, 2004.

11. Madhusudhan B, Rambhau D, Apte SS and Gopinath D: Oral bioavailability of flutamide from 1-o-alkylglycerol stabilized o/w nanoemulsions. J Disp Sci Technol 28: 1254-61, 2007.

12. Zeng X, Tao W, Mei L, Huang L, Tan C and Feng SS: Cholic acid-functionalized nanoparticles of star-shaped PLGA-vitamin E TPGS copolymer for docetaxel delivery to cervical cancer. Biomaterials 34: 6058-6067, 2013.

13. Thamake SI, Raut SL, Gryczynski Z, Ranjan AP and Vishwanatha JK: Alendronate coated poly-lactic-co-glycolic acid (PLGA) nanoparticles for active targeting of metastatic breast cancer. Biomaterials 33: 7164-7173, 2012.

14. Hrkach J, Von Hoff D, Ali MM, Andrianova E, Auer J, Campbell T, de Witt D, Figa M, Figueiredo M, Horhota A, et al: Preclinical development and clinical translation of a PSMA-targeted docetaxel nanoparticle with a differentiated pharmacological profile. Sci Transl Med 4: 128-39, 2012.

15. Ganju A, Yallapu MM, Khan S, Behrman SW, Chauhan SC and Jaggi M: Nanoways to overcome docetaxel resistance in prostate cancer. Drug Resist Updat 17: 13-23, 2014.

16. Hrkach J1, Von Hoff D, Mukkaram Ali M, Andrianova E, Auer J, Campbell T, De Witt D, Figa M, Figueiredo M, Horho ta A, et al. Preclinical development and clinical translation of a PSMA-targeted docetaxel nanoparticle with a differentiated pharmacological profile. Sci Transl Med 4: 128ra39, 2012

17. Yallapu MM, Gupta BK, Jaggi M and Chauhan SC. Fabrication of curcumin encapsulated PLGA nanoparticles for improved therapeutic effects in metastatic cancer cells. J Colloid Interface Sci 351: 19-29, 2010.

18. Kanfer I: Report on the international workshop on the biopharmaceutics classification system (BCS): Scientific and regulatory aspects in practice. J Pharm Pharm Sci 5: 1-4, 2002.

19. Le Y, Ji H, Chen JF, Shen Z, Yun J and Pu M: Nanosized bicalutamide and its molecular structure in solvents. Int $\mathrm{J}$ Pharm 370: 175-180, 2009.

20. Meer T, Fule R, Khanna D and Amin P. Solubility modulation of bicalutamide using porous silica. Int J Pharm Invest 43: 279-285, 2013

21. Sundaramoorthy P, Baskaran R, Mishra SK, Jeong KY, et al. Novel self-micellizing anticancer lipid nanoparticles induce cell death of colorectal cancer cells. Colloids Surf B Biointerfaces: Biointerfaces 135: 793-801, 2015.

22. Yallapu MM, Othman SF, Curtis ET, Gupta BK, Jaggi M and Chauhan SC: Multi-functional magnetic nanoparticles for magnetic resonance imaging and cancer therapy. Biomaterials 32: 1890-1905, 2011.

23. Solito E, de Coupade C, Canaider S, Goulding NJ and Perretti M. Transfection of annexin 1 in monocytic cells produces a high degree of spontaneous and stimulated apoptosis associated with caspase-3 activation. Br J Pharmacol 133: 217-228, 2001. 